\title{
Apport de l'embolisation de la varicocèle chez 51 patients infertiles
}

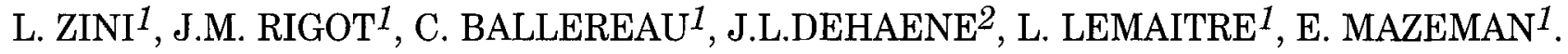 \\ ${ }^{1}$ CHRU Lille, ${ }^{2}$ EPARP Polyclinique du bois, Lille.
}

\section{RESUME}

L'objectif de cette étude a été d'analyser les modifications engendrées par la scléro-embolisation de la varicocèle sur la qualité du spermogramme de contrôle à 4 mois.

De février 93 à février 2000, 51 patients infertiles avec hypotrophie testiculaire ou oligospermie ont eu une embolisation de varicocèle. Tous ont bénéficié d'une échographie-doppler avec manoeuvre de Valsalva et d'un spermogramme. L'indication d'embolisation a été portée devant une oligo-asthéno-tératospermie ou une asymétrie testiculaire supérieure à $2 \mathrm{ml}$. Tous les patients ont été revus quatre mois plus tard avec un spermogramme.

Après embolisation, on a constaté la disparition clinique de la varicocèle dans $94 \%$ des cas (48 patients). La varicocèle persistait cliniquement ou échographiquement dans 2 cas. Dans $2 \%$ des cas ( 1 patient), il n'a pas été possible de cathétériser la veine spermatique gauche. L'évolution du spermogramme (valeurs moyennes) est la suivante : Sur un total de 50 patients ayant eu une embolisation, la numération de spermatozoïdes est passée de $19,6.106 / \mathrm{ml}$ à $26,7.106 / \mathrm{ml}$. Sur la population des 36 patients présentant une oligospermie ou une azoospermie $(<20.106 / \mathrm{ml})$ la numération est passée de $3.106 / \mathrm{ml}$ avant embolisation à $14,6.106 / \mathrm{ml}$ après embolisation. Pour le sous-groupe de 22 patients présentant une oligospermie entre 1.106 et $20.106 / \mathrm{ml}$ la numération est passée de $5,32.106 / \mathrm{ml}$ à $22,32.106 / \mathrm{ml}(\mathrm{p}<0.01) ;$ pour le sous-groupe de patients présentant une oligo- spermie sévère $(0<\mathrm{spz}<1.106 / \mathrm{ml})$ la numération est passée de $0,188.106 / \mathrm{ml}$ à $3,5.106 / \mathrm{ml}$ (p<0.01). Un des 4 patients présentant une azoospermie en pré-embolisation a eu une amélioration du spermogramme.

En conclusion, dans cette population d'hommes infertiles porteurs d'une varicocèle avec hypotrophie testiculaire, la scléro-embolisation permet d'améliorer l'oligospermie. Elle permet ainsi de proposer des inséminations intra-utérines ou des FIV en lieu et place de l'ICSI.

Mots clés : Infertilité, varicocèle, scléro-embolisation, spermogramme.

\section{INTRODUCTION}

La varicocèle est la dilatation variqueuse des veines du plexus pampiniforme. C'est une pathologie fréquente présente chez $15 \%$ de la population masculine, ayant un effet délétère sur la croissance du testicule et la spermatogenèse. Elle est considérée comme une cause réversible d'infertilité [16].

La base du traitement de la varicocèle est l'occlusion de la veine spermatique. De nom-

Correspondance : L. Zini, Service d'Urologie, Hôpital Huriez, CHRU de Lille, 59037 Lille cedex.

Communication orale sélectionnées au XVIIème Congrès de la SALF, 7-8 décembre 2000, Bordeaux. 
breuses techniques sont possibles. L'embolisation en radiologie interventionnelle est une alternative au traitement chirurgical. C'est une méthode efficace et réalisée en ambulatoire [8].

L'objectif de cette étude a été d'analyser les modifications engendrées par la scléro-embolisation sur le spermogramme de contrôle à 4 mois.

\section{MATÉRIEL ET MÉTHODES}

De février 1993 à février 2000, 51 patients infertiles avec hypotrophie testiculaire ou oligospermie ont eu une embolisation de varicocèle.

Tous ont eu avant embolisation un examen clinique par un seul consultant (Dr Rigot), une échographie couplée au doppler veineux avec manœuvre de Valsalva et au moins un spermogramme. L'échographie permettait une mesure objective et comparative des volumes testiculaires ainsi que la recherche de lésions infracliniques [5].

L'indication d'embolisation a été portée devant une oligospermie, une tératospermie ou une oligo-asthéno-tératospermie associée à une hypotrophie testiculaire.

Lintervention a été réalisée en ambulatoire, sous anesthésie locale, par ponction veineuse fémorale droite selon la technique de Seldinger. La phlébographie rénale et spermatique est le premier temps opératoire. Elle étudie la continence ostiale et la morphologie des veines spermatiques. Un cathétérisme sélectif sous amplificateur de brillance de la veine spermatique principale permet sa scléro-embolisation par l'injection d'un produit sclérosant (Aetoxisclérol®), polidocanol) et mise en place d'un stent métallique endo-vasculaire occlusif (Figure 1).

Tous les patients ont été revus en consultation quatre mois après l'intervention munis d'un spermogramme.

L'analyse statistique a été effectuée par le test de Student t. Une valeur de $\mathrm{p}<0.01$ était considérée comme statistiquement significative.

\section{RÉSULTATS}

Dans cette étude rétrospective l'âge moyen était de 31 ans (24 à $42 \mathrm{ans}$ ) et le délai moyen d'infertilité était de 30 mois (10 à 96 mois). Il s'agissait de 41 infertilités primaires et 10 secondaires. Dans cette série toutes les varicocèles étaient gauches et unilatérales. La varicocèle était palpable et non visible (grade 1) dans 12 cas, visible et palpable (grade 2) dans 31 cas et une grosse varicocèle (grade 3 ) dans 8 cas. Cette varicocèle était symptomatique (gêne fonctionnelle) dans 6 cas.

La volumétrie testiculaire en échographie notait une hypotrophie (inférieure à $16 \mathrm{ml}$ ) bilatérale dans $92 \%$ des cas ( 47 patients) et une asymétrie testiculaire avec une réduction $\mathrm{du}$ volume du testicule gauche par rapport au testicule droit supérieur à $2 \mathrm{ml}$ dans $57 \%$ des cas (29 patients).

La scléro-embolisation a permis une disparition de la varicocèle dans $94 \%$ des cas (48 patients). Dans un cas le cathétérisme de l'ostium de la veine spermatique gauche était impossible et dans deux cas la varicocèle persistait. Aucune complication n'a été notée.

L'évolution du spermogramme (Tableau 1) montre surtout une amélioration de la numération.

L'évolution de la numération en cas d'oligospermie (Tableau 2) était d'autant plus importante que l'oligospermie était sévère. En cas d'oligospermie avec une numération comprise entre $1.106 / \mathrm{ml}$ et $20.106 / \mathrm{ml}$ (22cas) l'augmentation était significative $(p<0.01)$. De même en cas d'oligospermie sévère comprise entre 0 et $1.106 / \mathrm{ml}$, l'augmentation de la numération était significative $(\mathrm{p}<0.01)$. La densité des spermatozoïdes a été multipliée au moins par trois dans $69,4 \%$ des cas d'oligospermie.

\section{DISCUSSION}

Seuls 15 à $20 \%$ des hommes qui présentent une varicocèle consultent pour infertilité. Mais une varicocèle est découverte chez $30 \%$ à $35 \%$ des patients présentant une infertilité primaire et chez plus de $80 \%$ des patients avec une infertilité secondaire $[6,4]$. De nombreuses 

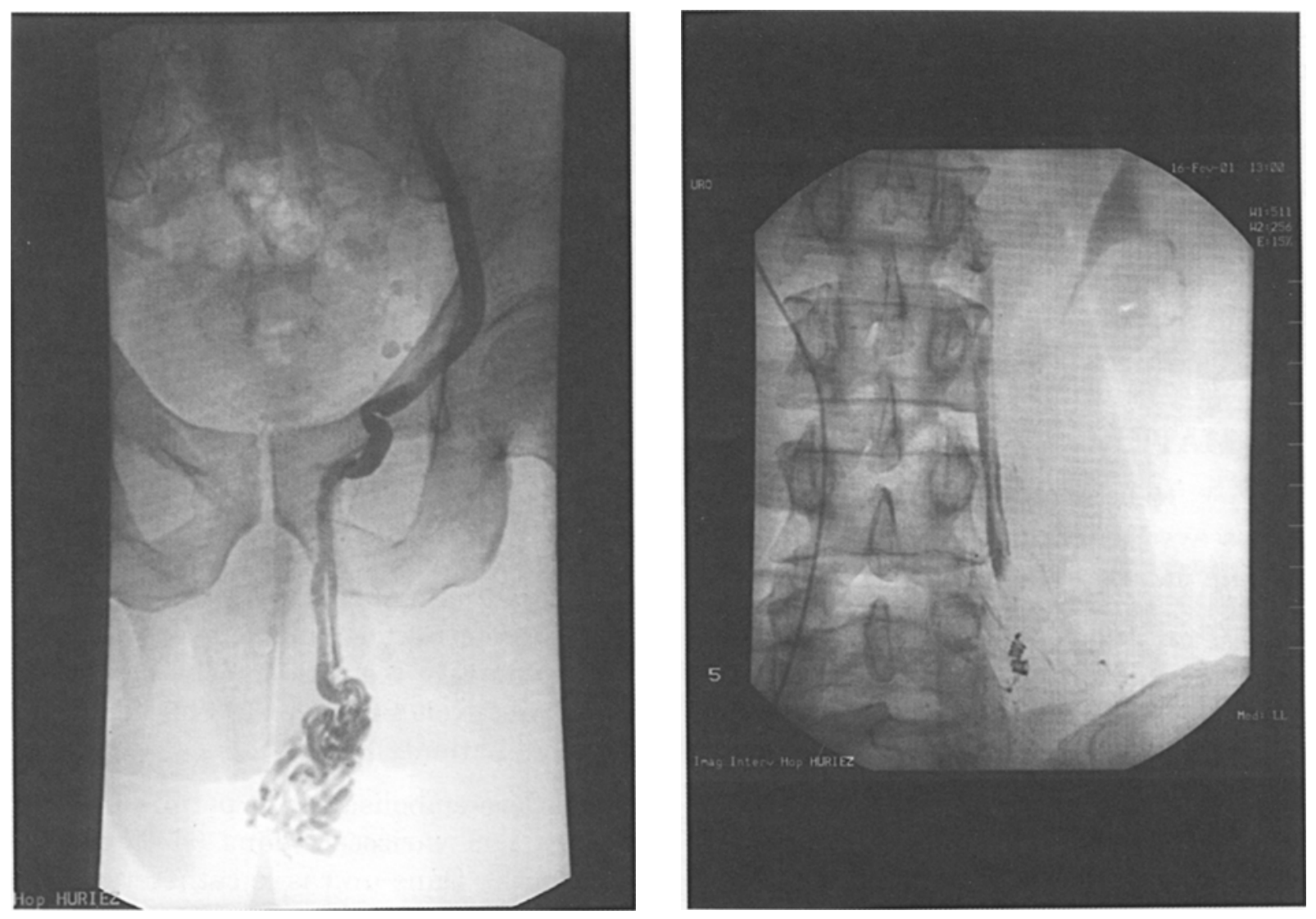

Figure 1 : Sclérose de la veine spermatique gauche après embolisation

Tableau 1: spermogramme avant et après embolisation

\begin{tabular}{lccc}
\hline & NuMÉRATION & MobILITÉ & TÉRATOSPERMIE \\
& $\mathbf{1 0 6} /$ ML & 3ÈME HeURE & $\%$ \\
& $\mathbf{5 0} \mathbf{~ C A S}$ & $\%$ & 72,4 \\
Avant embolisation & 19,6 & 17,9 & 67,4 \\
Après embolisation & 26,7 & 23 & \\
\hline
\end{tabular}

Tableau 2 : numération avant et après embolisation chez les patients oligospermiques.

\begin{tabular}{|c|c|c|c|c|c|}
\hline & $\begin{array}{l}\text { Oligo/Azoospermie } \\
<20.10^{6} / \mathrm{ml} \\
36 \mathrm{cas}\end{array}$ & $\begin{array}{c}\text { Oligospermie } \\
1.10^{6}<\mathrm{Spz}<20.106 / \mathrm{ml} \\
22 \mathrm{cas}\end{array}$ & $\begin{array}{c}\text { Oligospermie } \\
0<\mathrm{Spz}<1.106 / \mathrm{ml} \\
10 \mathrm{cas}\end{array}$ & $\begin{array}{c}\text { Azoospermie } \\
4 \text { cas }\end{array}$ & $\begin{array}{l}\text { Total } \\
\text { Embolisés } \\
50 \text { cas }\end{array}$ \\
\hline $\begin{array}{l}\text { Pré- } \\
\text { embolisation }\end{array}$ & $3.10^{6} / \mathrm{ml}$ & $5,32.10^{6} / \mathrm{ml}$ & $0,188.10^{6} / \mathrm{m}$ & 0 & $19,6.10^{6} / \mathrm{ml}$ \\
\hline Post-embolisation & n $\quad 14,6.10^{6} / \mathrm{ml}$ & $\begin{array}{c}23,32.10^{6} / \mathrm{ml} \\
\mathrm{p}<0,01\end{array}$ & $\begin{array}{c}3,5.10^{6} / \mathrm{ml} \\
\mathrm{p}<0.01\end{array}$ & 1 amélioré & $26,7.10^{6} / \mathrm{ml}$ \\
\hline
\end{tabular}


études ont montré que la varicocèle a un effet délétère sur la spermatogenèse avec en particulier une diminution de la densité des spermatozoïdes (oligospermie) et une diminution du pourcentage des formes mobiles (asthénospermie) $[2,9,3]$.

La majorité des auteurs s'accordent à dire que le traitement de la varicocèle permet d'améliorer la spermatogenèse et notamment la concentration et la mobilité des spermatozoïdes dans l'éjaculat $[14,7,13,15]$. Une étude prospective randomisée montre que la cure de varicocèle améliore les paramètres spermatiques [11].

La scléro-embolisation est une technique intéressante. Ces résultats sont bons et ses complications rares (réactions allergiques au produit de contraste) [10]. L'étude comparative des traitements possibles de la varicocèle ne montre pas de différence importante sur l'amélioration des paramètres spermatiques [1]. L'avantage de cette technique de radiologie interventionnelle est sa réalisation en ambulatoire et sous anesthésie locale.

Les résultats de notre étude vont dans le même sens avec surtout une amélioration de la concentration des spermatozoïdes même en cas d'oligospermie sévère inférieure à $1.106 / \mathrm{ml}$.

Cette approche thérapeutique des infertilités associées à une varicocèle permet une réduction du coût de la prise en charge des procréations médicalement assistées. Une étude récente du rapport coût/efficacité montre que la séquence cure de varicocèle puis fécondation in vitro (FIV) est meilleure que insémination intra-utérine (IIU) puis FIV [12].

\section{CONCLUSION}

Chez les hommes infertiles porteurs d'une varicocèle avec hypotrophie testiculaire, la scléro-embolisation permet d'améliorer de façon notable l'oligospermie. Elle pourrait ainsi modifier la technique de procréation médicalement assistée et permettrait de proposer une FIV voire une IIU en lieu et place de l'ICSI.

\section{RÉFÉRENCES}

1. BARBALIAS G.A., LIATSIKOS E.N., NIKIFORIDIS G., et al. : Treatement of varicocele for male infertility: a comparative study evaluating currently used approaches. Eur. Urol., 1998, 34 : 393-398.

2. CHEHVAL M.J., PURCELL M.H. : deteriorations of semen parameters over time in men with untreated varicocele: evidence of testicular damage. Fertil. Steril., 1992, 57 : 174-177.

3. FONTAINE E., BENOIT G., JARDIN A., BEURTON D. : La varicocèle de l'adolescent. Progrès en Urologie, 2000, $10: 1099-1107$.

4. GORELICK J., GOLDSTEIN M. : Loss infertility in men with varicocele. Fertil. Steril. 1993, 59 : 613-616.

5. GOULLET E., RIGOT J.M., BLOIS N., et al. : Intérêt de l'échographie scrotale systématique dans la prise en charge de l'homme infertile. Progrès en Urologie, $2000,10: 78-82$.

6. GREENBERG S.H., LIPSHULTZ L.I., WEIN A.J. : Experience with 425 subfertile male patients. J. Urol., 1978, $119:$ :507-510.

7. ISMAIL M.T., SEDOR J., IRSCH I.H. : Are sperm motion parameters influenced by varicocele ligation? Fertil. Steril., 1999, $71: 886-890$.

8. LIMA S.S., CASTRO M.P., COSTA O.F. : A new method for the treatment of varicocele. Andrologia, 1978, 10 : 103-106.

9. LIPSHULTZ L.I., CORRIERE J.N. Jr. : Progressive testicular atrophy in varicocele patient. J. Urol. 1977, $117: 175-176$.

10. LOPEZ C., SERRES-COUSINE O., AVEROUS M. : Varicocèle de l'adolescent. Traitement par sclérothérapie et embolisation percutanée. Progrès en Urologie, 1998, $8:$ 382-387.

11. MAGDAR I., WEISSENBERG R. LUNENEFELD B., et al. : Controlled trial of high spermatic vein ligation for varicocele in infertile men. Fertil. Steril., 1995, 63: 120-124.

12. PENSON D.F., PALTER S.J., PALTIEL A.D., et al. : Cost-effectiveness analysis of treatment strategies for varicocele-related infertility. J. Urol., 2000, 163, 4 (supplement) : 2-3.

13. SCHATTE E.C., HIRSHBERG S.J., FALLICK M.L., et al. : Varicocelectomy improves sperm strict morphology and motility. J. Urol., 1998, 160 : 1338-1340.

14. SCHLESINGER M.H., WILETS I.F., NAGLER H.M. : Treatement outcome after varicocelectomy. Urologic Clinics of North America, 1994 august, 21(3) : 517529.

15. SEFTEL D., RUTCHIK S.D., CHEN H., et al. : Effects of subinguinal varicocele ligation on sperm concentration, motility and Kruger morphology. J. Urol., 1997, $158: 1800-1803$.

16. TULLOCH W.S. : Varicocelectomy in subfertility : results of treatment. Br. Med.J., 1955, 2 : 356-358. 


\section{ABSTRACT}

Results of varicocele sclero-embolization in 51 infertile patients

L. ZINI, J.M. RIGOT, C. BALLEREAU, J.L.DEHAENE, L. LEMAITRE, E. MAZEMAN

This study evaluated the changes induced on the parameters of the sperm count four months after sclero-embolization of varicocele.

From February 1993 to February 2000, 51 infertile patients with testicular hypotrophy or oligospermia were treated by percutaneous sclero-embolization of varicocele. All patients underwent preoperative diagnostic evaluation by semen analysis, Doppler flowmetry or color Doppler ultrasound and venography. Scrotal ultrasound was performed to evaluate the echogenicity of the testes, their volume and funicular vein enlargement during Valsalva manoeuvre. Patients were reviewed four months after sclero-embolization with semen analysis.

Clinical follow-up showed complete resolution of the varicocele in $94 \%$ of cases $(47$ patients), while the varicocele persisted in two cases. In $2 \%$ of cases ( 1 patient), selective catheterization of the internal spermatic vein was impossible. Four months later, analysis of semen parameters showed an increased number of spermatozoa. In $69.4 \%$ of oligospermic cases, the sperm count was at least tripled. The time-course of the sperm count (average) was as follows: in a total of 50 patients with pre- and post-embolization evaluation, sperm count increased from $19.6106 / \mathrm{ml}$ to 26.7 $106 / \mathrm{ml}$. In the population of 36 patients with a sperm count $<20$ 106/ml (i.e. with oligospermia or azoospermia), the sperm count increased from $3106 / \mathrm{ml}$ to $14.6106 / \mathrm{ml}$. In the subgroup of patients with oligospermia $(1-20.106 / \mathrm{ml})$, the sperm count increased $(p<0.01$ ) from 5.32 $106 / \mathrm{ml}$ to $22.32106 / \mathrm{ml}$; in the subgroup of patients with severe oligospermia (0-1 $106 / \mathrm{ml})$, the sperm count increased $(\mathrm{p}<0.01)$ from $0.188106 / \mathrm{ml}$ to $3.5106 / \mathrm{ml}$. Semen analysis was improved in 1 of the 4 patients with azoospermia.

In conclusion, sclero-embolization improved sperm count in this population of infertile patients with varicocele and testicular hypotrophy. In vitro fertilization or intra-uterine insemination can therefore be considered in the place of ICSI.

Key words: infertility, varicocele, sclero-embolization, semen analysis. 